\title{
Bereitstellung von Spezialinstrumenten und Implantaten
}

\author{
S. Bäuerle
}

\section{Zusammenfassung}

Die Einbeziehung des OP-Personals in die präoperative Planung einer selten durchgeführten Operation gestaltet das aktuelle Geschehen positiv. Es sichert das Vorhandensein der benötigten Spezialinstrumente, der Implantate und die adäquate Einteilung der OP-Pflege für den Eingriff.

\section{Einleitung}

Die Information über die präoperative Planung einer Korrekturosteotomie ist für das Instrumentierpersonal sehr wichtig. Das Ziel aller Vorbereitung ist koordiniertes, wirtschaftliches und effektives Arbeiten. Eine gute Planung und Organisation fördert die Zufriedenheit aller Beteiligten am Operationsgeschehen und sichert den Ablauf. Die Motivation erhöht sich automatisch durch die Einbeziehung in den Operationsprozeß.

Ohne die Information startet die Vorbereitung mit einem Ratespiel, bezüglich der einzusetzenden Mittel. Der Schwierigkeitsgrad der Operation wird evtl. verkannt, da der schriftliche OP-Plan darüber nichts aussagt, und ein unerfahrener Mitarbeiter wird für die Operation eingeteilt.

Deshalb ist ein kurzer Anruf des Operateurs möglichst am Vortag mit Details der geplanten Operation so wirkungsvoll und unerläßlich! (Abb.1u.2)

Nicht vergessen;

OP informieren!

OP-JOURNAL 2000; 16: 98-99

(C) Georg Thieme Verlag Stuttgart · New York

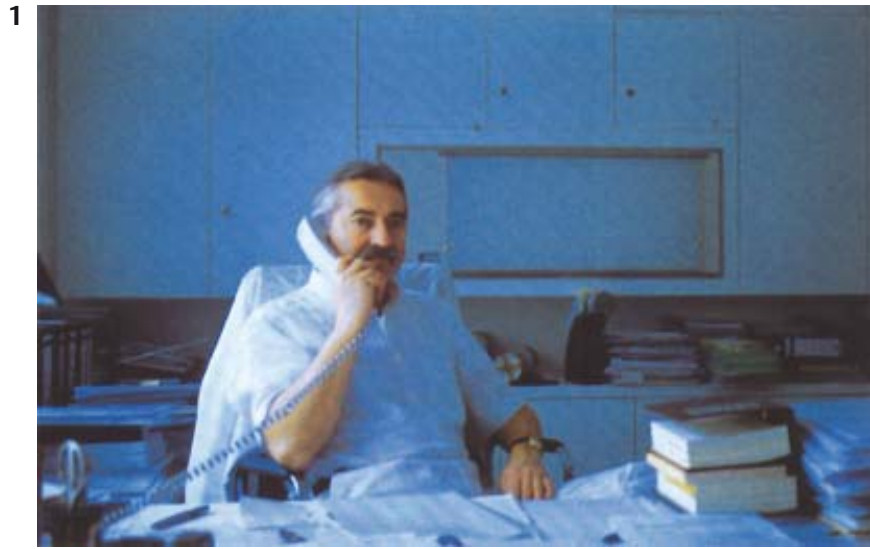

Abb. 1 u. 2

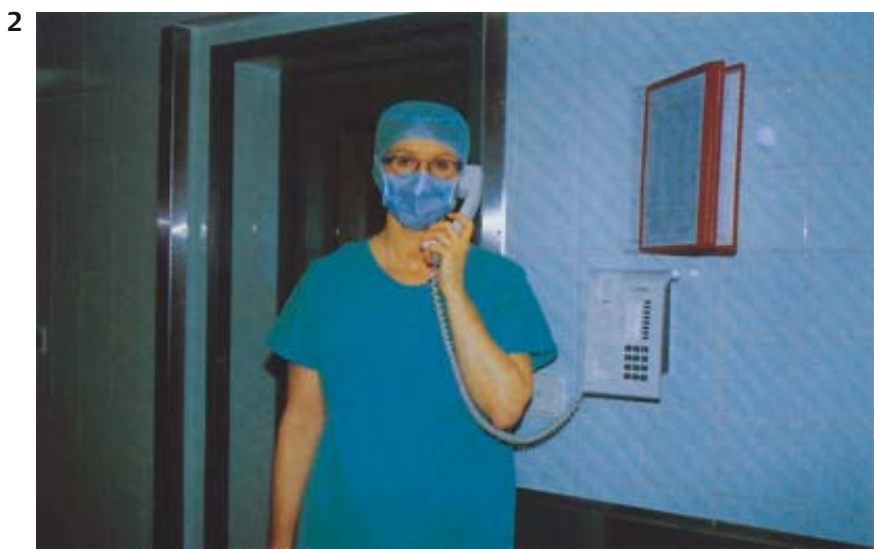

\section{Bereitstellung der Spezialinstrumente}

Meßlatte und Dreieckzielplatten

Die Meßlatte hilft die Plazierung der Osteotomie festzulegen. Die Dreieckzielplatten mit Gradangaben können zur Bestimmung der Winkel der Korrekturosteotomie verwendet werden (Abb. 3).

\section{Meißel}

Für die Sägeschnittführung der Osteotomie werden diverse Meissel bereitgestellt. Die Klingenmeißel mit scharfen, abgeschrägten Schneidekanten haben auswechselbare Klingen verschiedener Größe. Sie sind besonders grazil $(1 \mathrm{~mm})$ ähnlich wie auch die „Hand-Meissel“ und sind für feine Präparationen geeignet (Abb. 4).

Colibri

Zur Durchführung der präzisen Osteotomieschnitte eignet sich sehr gut die Antriebsmaschine Colibri. Da sie akkubetrieben ist, ist sie praktisch im Handling. Der oszillierende Sägeaufsatz ermöglicht ein unterschiedliches Positionieren der Sägeblätter. Es gibt ein umfangreiches Sägeblattsortiment mit einer Schnittdicke von nur 0,4 mm oder 0,6 mm (Abb.5).

Die Colibri-Aufsätze zum Bohren und Einbringen von Kirschner-Drähten kön- 


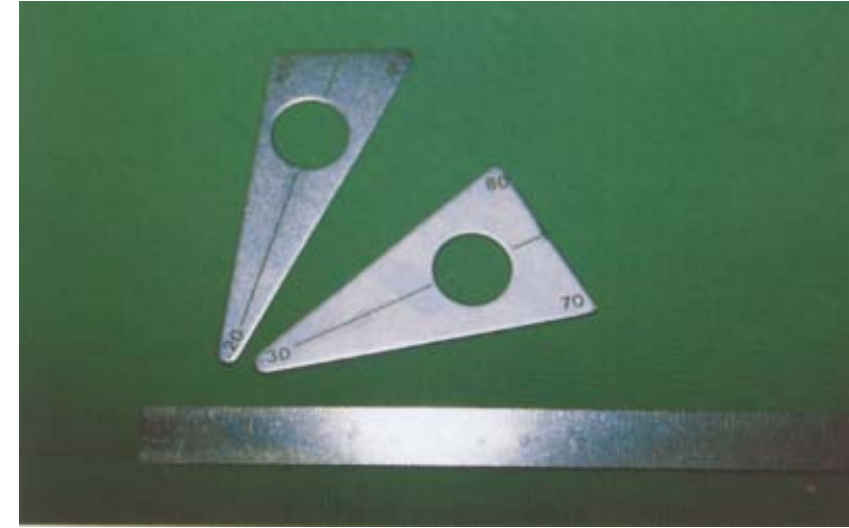

Abb. 3 Meßlatte und Dreieckzielplatten

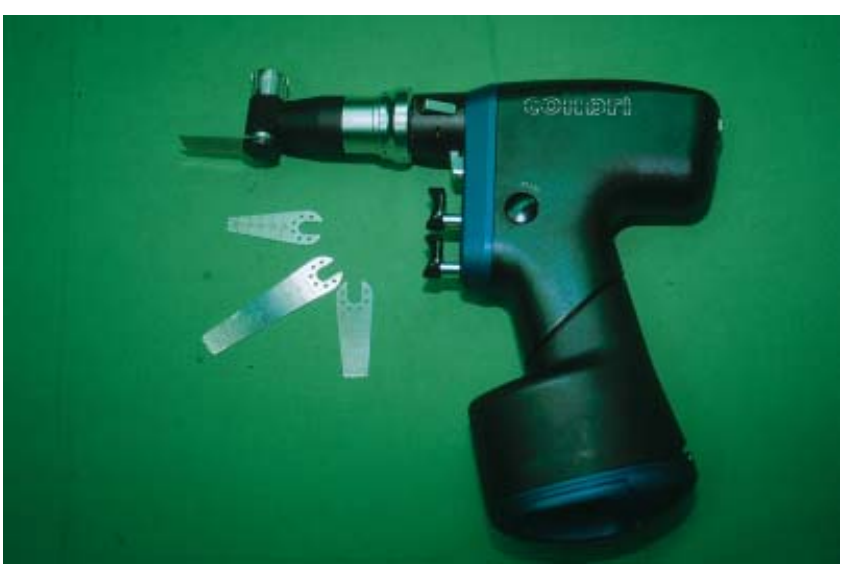

Abb.5 4 von 15 verschiedenen Sägeblättern mit oszillierender Säge

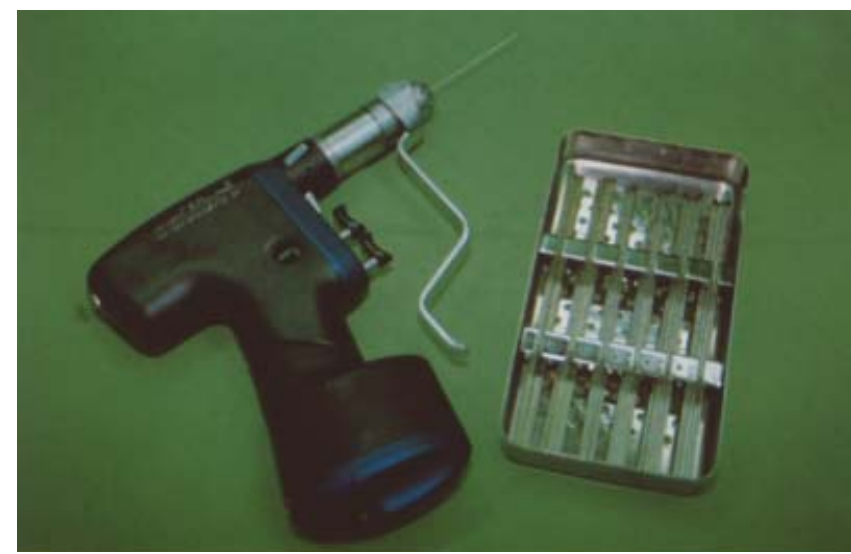

Abb.7 Colibri mit Schnellkupplung und Kirschner-Drahtsortiment

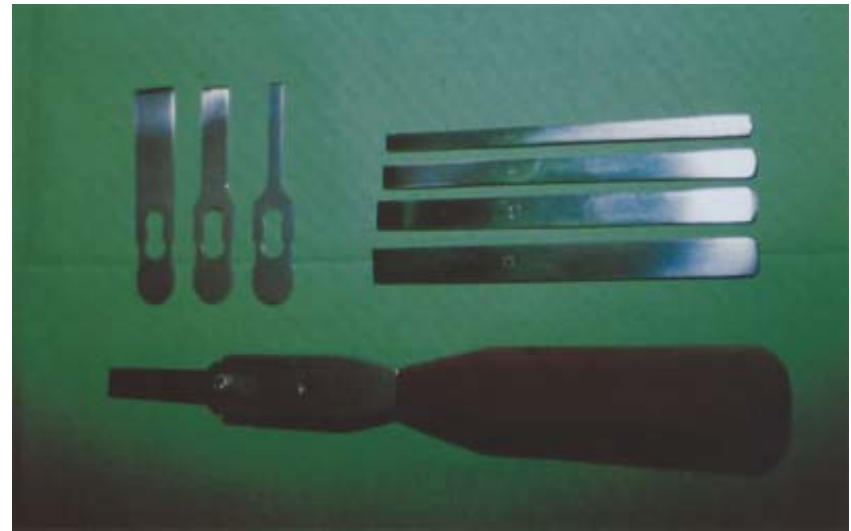

Abb. 4 Sortiment Klingenmeißel und Handmeißel

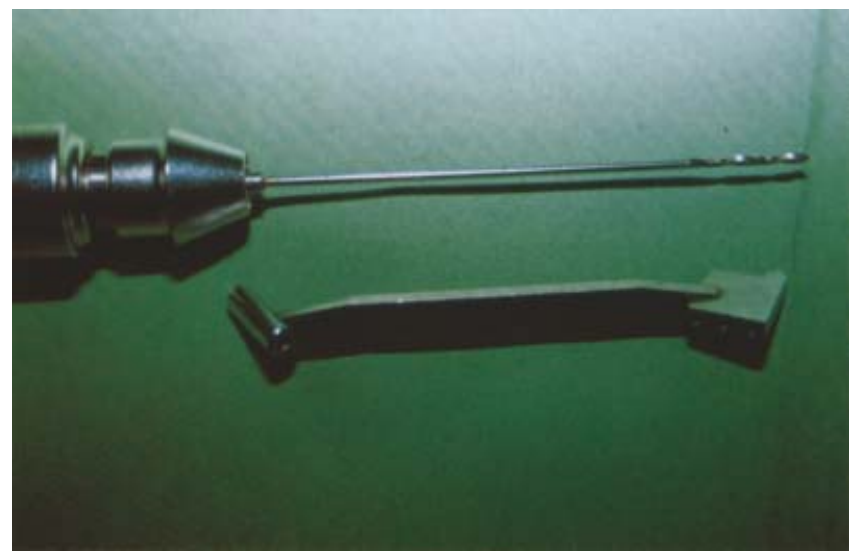

Abb. 6 2,0-mm-Bohrer mit Bohrhülse

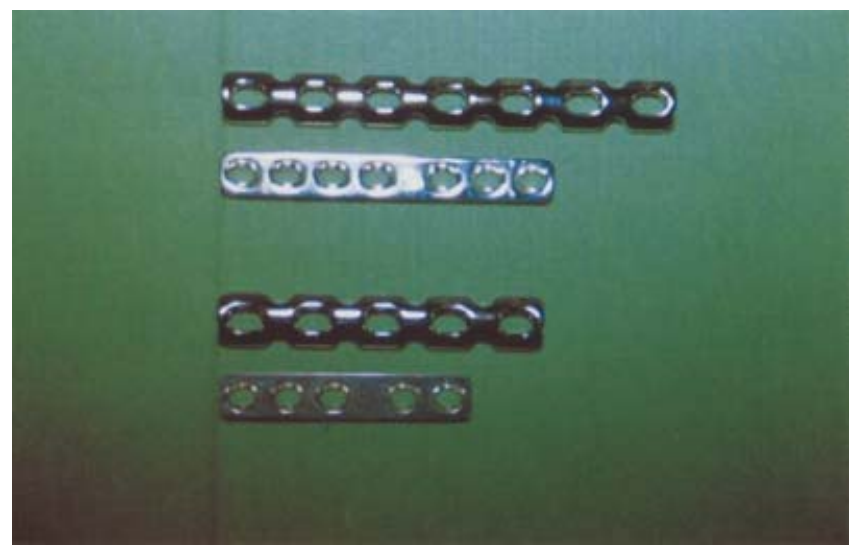

Abb. 8 2,7-mm-DC-Platte und 3,5-mm-Rekonstruktionsplatte gleicher Lochzahl nen bei der Korrekturosteotomie auch verwendet werden. Die mediale Kortikalis wird meist mit einem 2,0-mm-Bohrer geschwächt (Abb.6). Eine Auswahl an Kirschner-Drähten ist für die Winkelbestimmung und die Osteosynthese notwendig (Abb. 7).

\section{Bereitstellung der Implantate}

Die Implantatwahl richtet sich nach dem Alter des Patienten und der anatomischen Gegebenheit des Knochens. Die präoperative Planung entscheidet, ob die 2,7-mm-DC-Platten oder bzw. und die 3,5-mm-Rekonstruktionsplatten verwendet werden. Der Größenunterschied wird in Abb. 8 verdeutlicht.
Susanne Bäuerle

Stellv. Itd. OP-Schwester

BG Unfallklinik Tübingen

Schnarrenbergstr. 95

72076 Tübingen 